\section{(6)} OPEN ACCESS

\title{
Major incident triage and the implementation of a new triage tool, the MPTT-24
}

\author{
James Vassallo, ${ }^{1,2}$ J E Smith, ${ }^{3,4}$ L A Wallis ${ }^{1}$
}

\begin{abstract}
${ }^{1}$ Division of Emergency Medicine, University of Cape Town, Cape Town, South Africa ${ }^{2}$ Institute of Naval Medicine, Gosport, UK

${ }^{3}$ Emergency Department, Derriford Hospital, Plymouth, UK ${ }^{4}$ Academic Department of Military Emergency Medicine, Royal Centre for Defence Medicine (Research \& Academia), Medical Directorate, Birmingham, UK
\end{abstract}

\section{Correspondence to} Dr James Vassallo, Institute of Naval Medicine, Alverstoke, Gosport P012 2DL, UK; vassallo@doctors.org.uk

Received 1 June 2017 Revised 31 August 2017 Accepted 1 September 2017

Published Online First 21 October 2017

Check for updates

To cite: Vassallo J, Smith JE,
Wallis LA.
J R Army Med Corps
2018;164:103-106.

\section{ABSTRACT}

Introduction The Modified Physiological Triage Tool (MPTT) is a recently developed primary triage tool and in comparison with existing tools demonstrates the greatest sensitivity at predicting need for life-saving intervention (LSI) within both military and civilian populations. To improve its applicability, we proposed to increase the upper respiratory rate (RR) threshold to 24 breaths per minute (bpm) to produce the MPTT-24. Our aim was to conduct a feasibility analysis of the proposed MPTT-24, comparing its performance with the existing UK Military Sieve.

Method A retrospective review of the Joint Theatre Trauma Registry (JTTR) and Trauma Audit Research Network (TARN) databases was performed for all adult (>18 years) patients presenting between 2006-2013 (JTTR) and 2014 (TARN). Patients were defined as priority one (P1) if they received one or more LSIs. Using first recorded hospital RR in isolation, sensitivity and specificity of the $\geq 24 \mathrm{bpm}$ threshold was compared with the existing threshold ( $\geq 22 \mathrm{bpm}$ ) at predicting P1 status. Patients were then categorised as P1 or not-P1 by the MPTT, MPTT-24 and the UK Military Sieve.

Results The MPTT and MPTT-24 outperformed existing UK methods of triage with a statistically significant $(p<0.001)$ increase in sensitivity of between $25.5 \%$ and $29.5 \%$. In both populations, the MPTT-24 demonstrated an absolute reduction in sensitivity with an increase in specificity when compared with the MPTT. A statistically significant difference was observed between the MPTT and MPTT-24 in the way they categorised TARN and JTTR cases as P1 $(p<0.001)$.

Conclusions When compared with the existing MPTT, the MPTT-24 allows for a more rapid triage assessment. Both continue to outperform existing methods of primary major incident triage and within the military setting, the slight increase in undertriage is offset by a reduction in overtriage. We recommend that the MPTT-24 be considered as a replacement to the existing UK Military Sieve.

\section{INTRODUCTION}

Triage is the process of prioritising patients on the basis of their clinical acuity and is a key principle of effective major incident management. ${ }^{1}$ Within the UK, existing military and civilian doctrine utilises a two-stage approach to triage with primary and secondary triage being performed. Primary triage is a quick assessment of the patient, conducted at the scene and is frequently performed in difficult settings. For it to be effective, it must be rapid, reliable and reproducible, irrespective of the provider using it. ${ }^{1}$

The UK Military Sieve and National Ambulance Resilience Unit Sieve are the algorithms used by the

\section{Key messages}

The Modified Physiological Triage Tool (MPTT) was derived on a military cohort using logistic regression and outperforms all existing triage tools at predicting the need for life-saving intervention in both military and civilian populations.

- Increasing the upper respiratory rate threshold to 24 (MPTT-24) allows for a reduction in the time required to use the triage tool.

- Using the Alert; responds to Verbal stimulus; responds to Painful stimulus; Unresponsive (AVPU) scale as supposed to the GCS to measure conscious level will enable the MPTT-24 to be used by a greater number of personnel, increasing its applicability.

- Performance of the MPTT-24 is largely unchanged from the MPTT, and it clinically and statistically outperforms the existing UK Military Sieve at predicting the need for life-saving intervention.

- We recommend that the MPTT-24 be considered as an alternative to the existing UK Military Sieve for the purposes of primary major incident triage in the military setting.

Defence Medical Services and Ambulance Services, respectively, for primary major incident triage. ${ }^{23}$ Utilising simple physiological assessments, patients are categorised into one of three categories with priority one the most urgent. Secondary triage takes place in a more permissive environment, such as at a casualty clearing station or at the hospital entrance. Unlike primary triage, it is a more thorough assessment of the patient, frequently performed by more experienced and senior clinicians. If needed, it allows for the refinement of the triage category allocated during the primary triage process prior to treatment or admission to hospital. ${ }^{1}$

A number of studies have shown that existing methods of triage have limited accuracy at predicting the need for life-saving intervention in both the military and civilian environments. ${ }^{4}$ Derived specifically for this purpose, the Modified Physiological Triage Tool (MPTT) has shown the greatest sensitivity for predicting the need for lifesaving intervention, with the lowest rates of undertriage and acceptable levels of overtriage in both military and civilian populations. ${ }^{5-7}$

Respiratory Rate (RR) and Glasgow Coma Scale (GCS) form key components of the MPTT and can both be time consuming to accurately measure, 


\begin{tabular}{ll}
\hline & Intervention \\
\hline 1 & Intubation for actual airway obstruction \\
2 & Intubation for impending airway obstruction \\
3 & Surgical airway for airway obstruction \\
4 & Surgical airway for impending airway obstruction \\
5 & Needle thoracocentesis \\
6 & Finger thoracostomy \\
7 & Tube thoracostomy \\
8 & Application of a chest seal (commercial/improvised) \\
9 & Positive Pressure Ventilation for ventilatory inadequacy \\
10 & Application of a tourniquet for haemorrhage control \\
11 & Use of haemostatic agents for haemorrhage control \\
12 & Insertion of an intra-osseous device for resuscitation purposes \\
13 & Receiving uncross-matched blood \\
14 & Receiving $\geq 4$ units of blood/blood products \\
15 & Administration of tranexamic acid \\
16 & Laparotomy for trauma \\
17 & Thoracotomy for trauma \\
18 & Pericardial window for trauma \\
19 & Surgery to gain proximal vascular control \\
20 & Interventional radiology for haemorrhage control \\
21 & Application of a pelvic binder \\
22 & ALS/ACLS protocols for a patient in a peri-arrest situation \\
23 & ALS/ACLS protocols for a patient in cardiac arrest \\
24 & Neurosurgery for the evacuation of an intra-cranial haematoma \\
25 & Craniotomy \\
26 & Burr Hole Insertion \\
27 & Spinal nursing for a C1-3 fracture \\
28 & Administration of a seizure-terminating medication \\
29 & Active rewarming for initial core temp $<32^{\circ}$ celcius \\
30 & Passive rewarming for initial core temp $<32^{\circ}$ celcius \\
31 & Correction of low blood glucose \\
32 & Administration of chemical antidotes \\
\hline &
\end{tabular}

Figure 1 Life-saving interventions defining the priority one patient. ACLS, advanced cardiovascular life support.

with significant inter-rater reliability being described previously. $^{8}$ We propose to modify the MPTT by increasing the upper respiratory rate threshold to 24 breaths per minute (MPTT-24), allowing providers to more easily do a 15 second RR count and multiply by four (or 10 seconds and multiply by six), thereby potentially halving the time currently required to use the MPTT. In addition, we have adopted the Alert; responds to Verbal stimulus; responds to Painful stimulus; Unresponsive (AVPU) scale for the purposes of the conscious level assessment, replacing the existing GCS $<14$ assessment. ${ }^{9} 10$

Accepting a more pragmatic approach-with a threshold RR which is easily calculated within a shorter time frame-may change the test characteristics of the MPTT. The aim of this study was to conduct a feasibility analysis of the proposed MPTT-24 and compare its test characteristics with both the original MPTT and the existing UK Military Sieve.

\section{MATERIALS AND METHODS}

A retrospective review of the Joint Theatre Trauma Registry (JTTR) and Trauma Audit Research Network (TARN) databases was performed for all adult ( $\geq 18$ years) patients presenting between 2006-2013 (JTTR) and 2014 (TARN).

The JTTR holds data on all seriously injured patients treated by UK Defence Medical Services in the deployed setting with continuous data available from 2003. The default entry criterion was a patient who triggered trauma team activation, but this was expanded in 2007 to include all patients with trauma who were returned to the Royal Centre for Defence Medicine for definitive treatment. ${ }^{511}$ Established in 1988, TARN is the largest trauma database in Europe, collecting data from all trauma receiving hospitals in England and Wales on patients with moderate to severe injuries and contains data from point of injury through to discharge. TARN inclusion criteria include hospital admission $>3$ days, admission to critical care or death in hospital. ${ }^{12} 13$ Patients declared dead at scene and not conveyed to hospital are not included in the database. Patients were assumed to be non-ambulant due to the nature of the TARN database and its inclusion criteria. ${ }^{6}$

Patients were defined as priority one (P1) if they had received one or more life-saving interventions from a previously defined list, derived through international consensus of experts involved in major incident management (Figure 1). ${ }^{14}$ Using first recorded hospital RR in isolation, the sensitivity and specificity of the $\geq 24$ breaths per minute threshold were compared with the existing threshold ( $\geq 22$ breaths per minute) at predicting P1 status. Patients were then categorised as P1 or not-P1 by the MPTT, the MPTT-24 (Figure 2) and the UK Military Sieve. A McNemar test was used to determine statistical significance between the triage tools.

\section{ETHICS STATEMENT}

The use of the JTTR was approved by the Medical Directorate, Royal Centre for Defence Medicine. Additionally, as part of a larger programme of work, this study received ethical approval from the Human Research Ethics Committee of the University of Cape Town, the primary academic institution of the lead author (reference 285/2013).

\section{RESULTS}

Basic study characteristics are shown in table 1 . In both populations, the increased threshold in RR in isolation was associated with an absolute reduction in sensitivity (TARN 11.4\%, JTTR 13.5\%) and an increase in specificity (TARN 8.9\%, JTTR 13.8\%). An increase in OR and positive predictive value was observed when using a higher RR for both TARN and JTTR (table 2).

When incorporated into the MPTT-24, an absolute reduction in sensitivity was observed (TARN 8.9\%, JTTR 3.2\%) with an increase in specificity. There was a statistically significant difference between the MPTT and MPTT-24 in the way they categorised TARN and JTTR cases as P1 ( $<00.001)$.

The MPTT-24 demonstrated a statistically significant $(\mathrm{p}<0.001)$ increase in sensitivity (TARN 25.5\%, JTTR 23.5\%) over the existing UK Military Sieve in its ability to identify those in need of a life-saving intervention.

\section{DISCUSSION}

In this study, we have demonstrated that pragmatic modifications to the MPTT, in the form of the MPTT-24, can be implemented while maintaining comparable performance at predicting the need for life-saving intervention in both civilian and military trauma registry populations. With these modifications, the MPTT-24 continues to outperform the existing UK Military Sieve.

In keeping with the existing UK Military Sieve, ${ }^{2}$ we have included an assessment of catastrophic external haemorrhage in the MPTT-24. While experience of such injuries is likely to be limited in day-to-day civilian trauma care, we note previous European terrorist major incidents (Paris 2015 and London 2007) where the demand for tourniquets was high. ${ }^{15}$ In the context of an ongoing Marauding Terrorist Firearms Attack, the ability to provide treatment will be limited; controlling catastrophic external haemorrhage through tourniquets or haemostatic dressings may help to preserve life until the incident becomes more permissive.

The MPTT assesses the patient's conscious level using the GCS, with patients scoring 13 or lower being considered P1. While this represents the optimum threshold of conscious level at predicting need for life-saving intervention, it is not 


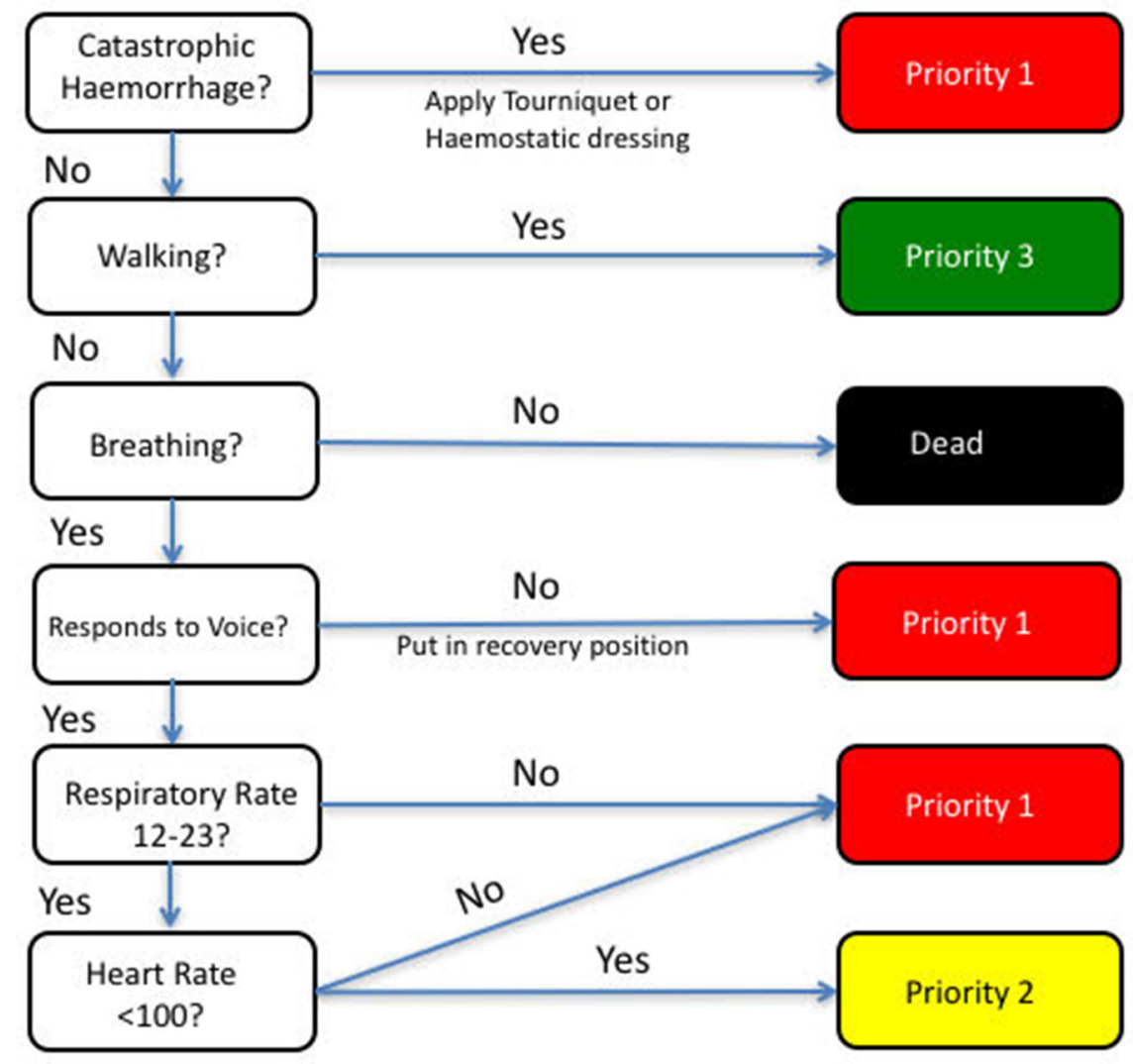

Figure 2 Modified Physiological Triage Tool (MPTT)-24 algorithm with increased respiratory rate upper threshold ( $\geq 24$ ), conscious level assessment using Alert; responds to Verbal stimulus; responds to Painful stimulus; Unresponsive (AVPU scale) and the additional assessment for external catastrophic haemorrhage. Vassallo 2017. CC BY 4.0.

\begin{tabular}{|c|c|c|}
\hline & JTTR (2006-2013) & TARN (2006-2014) \\
\hline Number of cases & 3654 & 127233 \\
\hline Male $\mathrm{N}, \%$ & $3593(98.3 \%)$ & 70747 (55.6\%) \\
\hline Age, median (IQR) & $24(21-29)$ & $61.4(43.1-80.0)$ \\
\hline ISS, median (IQR) & $5(2-16)$ & $9(9-16)$ \\
\hline Mortality & $2.1 \%$ & $5.7 \%$ \\
\hline Mechanism of injury $\mathrm{N}, \%$ & $\begin{array}{l}\text { Explosive 2012, 55.1\% } \\
\text { GSW 1252, 34.3\% }\end{array}$ & $\begin{array}{l}\text { Fall }<2 \text { m } 18 \text { 141, } 14.3 \% \\
\text { RTC } 27 \text { 915, } 21.9 \%\end{array}$ \\
\hline Injured body region $\mathrm{N}, \%$ & $\begin{array}{l}\text { Lower limb 1317, 36.0\% } \\
\text { Upper limb 593, 16.2\% }\end{array}$ & $\begin{array}{l}\text { Limb } 43 \text { 989, 34.6\% } \\
\text { Head } 24 \text { 732, 19.4\% }\end{array}$ \\
\hline Priority One $\mathrm{N}, \%$ & $1738,47.6 \%$ & $24791,19.5 \%$ \\
\hline
\end{tabular}

GSW, Gun shot wound; JTTR, Joint Theatre Trauma Registry; RTC, Road traffic collision; TARN, Trauma Audit Research Network.

without limitations. ${ }^{5}$ Previous studies have demonstrated wide inter-rater reliability when using the GCS. ${ }^{8}$ Calculating the GCS requires familiarity and prior experience with the scale, but even then it can be time consuming. The AVPU score was designed as a rapid assessment of conscious level and is a simpler alternative to the GCS. ${ }^{16}{ }^{17}$ A number of studies have looked at the correlation between GCS and AVPU with agreement that the division between being 'alert' and 'responds to voice' occurs at a median GCS of 13. ${ }^{916} 18$ For the purposes of simplifying the conscious level assessment in the primary triage process, we have replaced 'GCS $<14$ ' with 'responds to voice' in the MPTT-24. This pragmatic step should allow users with limited medical training to be able to use the MPTT-24 with similar results, thus increasing both its usability and applicability in the major incident setting.

A key principle of primary major incident triage is that it can be conducted rapidly and measuring the respiratory rate can be time consuming. By increasing the upper respiratory rate threshold of the MPTT, users are able to measure the respiratory rate over a 15 second period, allowing for a potential reduction in the time required to prioritise patients with the MPTT-24 by up to 15 seconds. If this reduction is applied to a theoretical scenario with 20 patients requiring triage, then up to 5 min could be saved by using the MPTT-24 rather than the MPTT. However, we acknowledge that this increased threshold is unlikely to convey any additional time benefit if users choose to measure the respiratory rate over a 30 second period.

Adopting the MPTT-24 comes at the expense of a reduction in sensitivity and therefore a higher rate of undertriage (1 - sensitivity). Clinically, this increased rate in undertriage is negligible; within the military setting, 30 genuine P1 patients would need to be assessed before an additional patient is undertriaged by the MPTT-24. Likewise, the reduction in overtriage (1 - positive predictive value)is negligible between the MPTT and MPTT-24 and needs even greater number of patients before a difference is observed.

In the civilian setting, the rate of overtriage for both the MPTT-24 and MPTT is high (66.0\% and $67.1 \%$, respectively). Although this is comparable to the overall overtriage rate following the London $7 / 7$ attacks (64\%), ${ }^{19}$ we acknowledge that if sustained, this level may not be tolerable in the setting of a non-developed system or rural environment. ${ }^{20}$ The MPTT and 
Table 2 Performance analysis of test characteristics for RR ( $\geq 22$ and 24 thresholds), MPTT, MPTT-24 and the existing UK Military Sieve

\begin{tabular}{lllll}
\hline & Sensitivity & Specificity & OR & PPV \\
\hline JTTR & & & & \\
RR $\geq 22$ & $48.7 \%(45.5-51.9)$ & $68.2 \%(65.1 \%-71.2 \%)$ & $2.04(1.69-2.45)$ & $61.1 \%(65.2-71.2)$ \\
RR $\geq 24$ & $35.2 \%(32.2-38.4)$ & $82.0 \%(79.4 \%-84.4 \%)$ & $2.48(2.01-3.07)$ & $66.7 \%(62.5-70.7)$ \\
MPTT & $69.9 \%(67.7-72.0)$ & $65.3 \%(63.2-67.4)$ & $4.37(1.90-5.02)$ & $64.8 \%(62.7-67.0)$ \\
MPTT-24 & $66.7 \%(64.5-68.9)$ & $69.9 \%(67.8-71.9)$ & $4.65(2.02-5.34)$ & $67.0 \%(64.7-69.1)$ \\
UK Military Sieve & $43.2 \%(40.9-45.6)$ & $93.7 \%(92.5-94.7)$ & $11.29(9.18-13.88)$ & $86.1 \%(83.6-88.4)$ \\
TARN & & & $2.56(2.47-4.16)$ & $35.6 \%(35.0-36.3)$ \\
RR $\geq 22$ & $47.5 \%(46.7-48.3)$ & $73.9 \%(73.6-74.3)$ & $2.73(1.31-2.84)$ & $39.0 \%(38.3-39.8)$ \\
RR $\geq 24$ & $36.1 \%(35.4-36.9)$ & $82.8 \%(82.5-83.2)$ & $3.41(3.31-3.51)$ & $32.9 \%(32.4-33.2)$ \\
MPTT & $57.8 \%(56.9-58.2)$ & $71.5 \%(71.3-71.8)$ & $3.43(3.33-3.53)$ & $34.0 \%(33.4-34.5)$ \\
MPTT-24 & $53.5 \%(52.9-54.1)$ & $74.8 \%(74.6-75.1)$ & $6.17(5.94-6.41)$ & $53.3 \%(52.5-54.2)$ \\
\hline UK Military Sieve & $28.0 \%(27.5-28.6)$ & $94.1 \%(93.9-94.2)$ & \\
\hline
\end{tabular}

JTTR, Joint Theatre Trauma Registry; RR, Respiratory Rate; MPTT, Modified Physiological Triage Tool; PPV, positive predictive value; TARN, Trauma Audit Research Network.

MPTT-24 were designed for the purposes of primary major incident triage alone and not as a replacement to secondary triage. Within the UK, patients will undergo a secondary triage process, with a review of the original triage categories and where appropriate, those initially overtriaged can be reallocated to a lower triage category at the discretion of experienced clinicians, thus reducing the overall overtriage rate. ${ }^{1}$

A key limitation of our study is the use of first recorded hospital physiology to calculate triage priorities. While prehospital data is recorded on both the JTTR and TARN databases, complete data are available for only $16.7 \%$ and $37.2 \%$ of military and civilian cases, respectively, making prioritisation with prehospital data unreliable. However, when complete hospital and prehospital physiology were compared in both datasets, we observed that the median and IQR were almost identical.

\section{CONCLUSION}

When compared with the existing MPTT, the MPTT-24 allows for the potential for a more rapid triage assessment, while maintaining comparable performance and continuing to outperform existing methods used in the UK. Within a military setting, the slight increase in undertriage is offset by a reduction in overtriage. We recommend that the MPTT-24 be considered as a replacement to the existing UK Military Sieve for the purposes of primary major incident triage.

Correction notice This article has been corrected since it was published Online First. Figure 2 has been corrected.

Twitter@jamievassallo

Acknowledgements Our thanks to Professor Fiona Lecky, Research Director and Antoinette Edwards, Chief Executive Officer, at the Trauma Audit and Research Network (TARN) for facilitating access to the TARN database.

Contributors JV conceived the study and conducted the analysis supervised by JES. JV drafted the manuscript and all authors contributed substantially to its revision. JV takes the responsibility for the paper as a whole.

Competing interests JV and JES are serving members of HM Armed Forces.

Provenance and peer review Not commissioned; externally peer reviewed.

Open Access This is an Open Access article distributed in accordance with the terms of the Creative Commons Attribution (CC BY 4.0) license, which permits others to distribute, remix, adapt and build upon this work, for commercial use, provided the original work is properly cited. See: http://creativecommons.org/licenses/by/4.0/

(c) Article author(s) (or their employer(s) unless otherwise stated in the text of the article) 2018. All rights reserved. No commercial use is permitted unless otherwise expressly granted.

\section{REFERENCES}

1 Advanced Life Support Group. Triage. In: Carley S, Mackway-Jones K, Major incident medical management and support. Oxford, UK: Blackwell Publishing Ltd, 2005:97-107.

2 Ministry of Defence. Clinical guidelines for operations (JSP 999). 2013 https://www. gov.uk/government/publications/jsp-999-clinical-guidelines-for-operations

3 National Ambulance Resilience Unit, United Kingdom. NARU input to new triage sieve. 2014. Cited 26 May 2017 https://naru.org.uk/naru-input-to-new-triage-sieve/

4 Challen K, Walter D. Major incident triage: comparative validation using data from 7th July bombings. Injury 2013;44:629-33.

5 Vassallo J, Beavis J, Smith JE, et al. Major incident triage: derivation and comparative analysis of the Modified Physiological Triage Tool (MPTT). Injury 2017;48:992-9.

6 Vassallo J, Smith J, Bouamra 0, et al. The civilian validation of the Modified Physiological Triage Tool (MPTT): an evidence-based approach to primary major incident triage. Emerg Med J 2017 (accessed 23 Jul 2017) [Epub ahead of print].

7 Vassallo J, Horne S, Smith JE, et al. The prospective validation of the Modified Physiological Triage Tool (MPTT): an evidence-based approach to major incident triage. J R Army Med Corps 2017;163:383-7.

8 Gill MR, Reiley DG, Green SM. Interrater reliability of Glasgow Coma Scale scores in the emergency department. Ann Emerg Med 2004:43:215-23.

9 Kelly CA, Upex A, Bateman DN. Comparison of consciousness level assessment in the poisoned patient using the alert/verbal/painful/unresponsive scale and the Glasgow Coma Scale. Ann Emerg Med 2004;44:108-13.

10 Mackay CA, Burke DP, Burke JA, et al. Association between the assessment of conscious level using the AVPU system and the Glasgow Coma Scale. Pre-hospital Immediate Care 2000:17-19.

11 Smith JE. The epidemiology of blast lung injury during recent military conflicts: a retrospective database review of cases presenting to deployed military hospitals, 2003-2009. Philos Trans R Soc Lond B Biol Sci 2011;366:291-4.

12 Kehoe A, Smith JE, Edwards A, et al. The changing face of major trauma in the UK. Emerg Med J 2015;32:911-5.

13 Barnard EB, Morrison JJ, Madureira RM, et al. Resuscitative Endovascular Balloon Occlusion of the Aorta (REBOA): a population based gap analysis of trauma patients in England and Wales. Emerg Med J 2015;32:926-32.

14 Vassallo J, Smith JE, Bruijns SR, et al. Major incident triage: a consensus based definition of the essential life-saving interventions during the definitive care phase of a major incident. Injury 2016;47:1898-902.

15 Hirsch M, Carli P, Nizard R, et al. The medical response to multisite terrorist attacks in Paris. Lancet 2015;386:2535-8.

16 Gill M, Martens K, Lynch EL, et al. Interrater reliability of 3 simplified neurologic scales applied to adults presenting to the emergency department with altered levels of consciousness. Ann Emerg Med 2007;49:403-7.

17 Kheirabadi AR, Tabeshpour J, Afshari R. Comparison of three consciousness assessment scales in poisoned patients and recommendation of a new scale: AVPU plus. Asia Pac J Med Toxicol 2015;4:58-63.

18 McNarry AF, Goldhill DR. Simple bedside assessment of level of consciousness: comparison of two simple assessment scales with the Glasgow Coma scale. Anaesthesia 2004:59:34-7.

19 Aylwin CJ, König TC, Brennan NW, et al. Reduction in critical mortality in urban mass casualty incidents: analysis of triage, surge, and resource use after the London bombings on July 7, 2005. Lancet 2006;368:2219-25.

20 Frykberg ER. Medical management of disasters and mass casualties from terrorist bombings: how can we cope? J Trauma 2002;53:201-12. 